Tropical Journal of Pharmaceutical Research October 2017; 16 (10): 2315-2324

ISSN: $1596-5996$ (print); 1596-9827 (electronic)

(C) Pharmacotherapy Group, Faculty of Pharmacy, University of Benin, Benin City, 300001 Nigeria.

All rights reserved.

Available online at http://www.tjpr.org

Original Research Article

http://dx.doi.org/10.4314/tjpr.v16i10.2

\title{
Comparative assessment of poly (D,L-lactide-co-glycolide) nanoparticles modified by either cetyltrimethylammonium bromide or chitosan for plasmid DNA adsorption
}

\author{
Abd Almonem Doolaanea,2*, Nur 'Izzati Mansor', Nurul Hafizah Mohd Nor', \\ Mohd Affendi Bin Mohd Shafri ${ }^{3}$, Susi Sukmasari ${ }^{4}$ and Farahidah Mohamed ${ }^{1,2,5}$ \\ ${ }^{1}$ Department of Pharmaceutical Technology, ${ }^{2} I K O P$ Sdn. Bhd., Pilot Plant Pharmaceutical Manufacturing, Faculty of Pharmacy, \\ ${ }^{3}$ Kulliyyah of Allied Health Sciences, ${ }^{4}$ Kulliyyah of Dentisry, International Islamic University Malaysia (IIUM), 25200 Kuantan, \\ ${ }^{5}$ International Institute of Halal Research \& Training (INHART), Kulliyyah of Engineering, IIUM, PO Box 10, 50728, Kuala \\ Lumpur, Malaysia
}

*For correspondence: Email: abdalmonemdoolaanea@yahoo.com; Tel: +60136238628; Fax: +609 5716775

Sent for review: 7 May 2017

Revised accepted: 23 September 2017

\begin{abstract}
Purpose: To evaluate poly (D,L-lactide-co-glycolide) PLGA nanoparticles modified by cetyltrimethyl ammonium bromide (CTAB) or chitosan for plasmid DNA adsorption.

Methods: PLGA nanoparticles were prepared by solvent diffusion method and modified by including CTAB in the aqueous (F1) or oil phase (F2), or by including low (F3) or medium (F4) molecular weight chitosan. The nanoparticles were characterised by differential scanning calorimetry (DSC) and Fourier transform infrared spectroscopy (FTIR), as well as for cell toxicity, cell uptake and transfection.

Results: CTAB failed to confer positive charge on the nanoparticles. CTAB desorbed easily from F1 surface. This resulted in negative zeta potential, increased cytotoxicity as well as decreased cell uptake and transfection. In F2, CTAB was located mainly in PLGA matrix, resulting in negative charge with decreased cytotoxicity, and increased cell uptake and transfection compared to F1. On the other hand, chitosan-modified nanoparticles (F3 and F4) showed stronger interaction between chitosan and PLGA, leading to positively-charged particles, decreased cytotoxicity, as well as increased cell uptake and transfection. Amongst the four formulations, F4 exhibited the highest transfection.

Conclusion: These results should aid in understanding how PLGA nanoparticles are modified by CTAB and chitosan. Modification with chitosan yields PLGA nanoparticles with higher DNA adsorption and transfection with lower cytotoxicity.
\end{abstract}

Keywords: Chitosan, cetyltrimethyl ammonium bromide (CTAB), Nanoparticle, Poly (D,L-lactide-coglycolide) PLGA, Plasmid DNA adsorption, Gene therapy

Tropical Journal of Pharmaceutical Research is indexed by Science Citation Index (SciSearch), Scopus, International Pharmaceutical Abstract, Chemical Abstracts, Embase, Index Copernicus, EBSCO, African Index Medicus, JournalSeek, Journal Citation Reports/Science Edition, Directory of Open Access Journals (DOAJ), African Journal Online, Bioline International, Open-J-Gate and Pharmacy Abstracts

\section{INTRODUCTION}

Poly (D, L-Lactic-co-Glycolic Acid) polymer (PLGA) is biodegradable and biocompatible polymer widely used in microencapsulation. Its robust synthetic pathway allows constructing variety of sustained release drug delivery devices $[1,2]$. PLGA nanoparticles and microparticles have been frequently used to deliver nucleic acids after being modified to become positively charged [3]. The positively charged particles interact with the negatively charged nucleic acids by means of electrostatic interactions to make a stable complex that can protect and deliver DNA or RNA. Nanoparticles with positive charge are more effective than negatively charged ones in 
the lysosomal escape [4]. Some of the commonly used surface modifying materials include chitosan [3] and cetyltrimethylammonium bromide (CTAB) [5].

Chitosan is a natural polymer (macromolecule) found to confer positive charge onto PLGA nanospheres [6]. A positive charge of $10 \mathrm{mV}$ at $\mathrm{pH} 7.4$ was sufficient to efficiently complex DNA electrostatically. PLGA nanoparticles for gene therapy need to be taken by cells first to exhibit their efficacy. Chitosan coating was found to enhance the cell uptake of PLGA nanoparticles compared to un-coated nanoparticles [7]. Chitosan-PLGA nanoparticles were found also to protect plasmid DNA from enzymatic degradation, which is important for clinical applications [8].

On the other hand, CTAB is small molecule employed as a cationic modifier for PLGA nanoparticles in several studies. [9] found that $\mathrm{CTAB}$, added to aqueous phase during fabrication, remained mainly associated with PLGA nanoparticles and ready for DNA adsorption, which is in turn associated with CTAB content in the nanoparticles. Subsequently, the authors modified their preparation method to include CTAB in aqueous phase at small concentration that was not needed to do a washing step [10]. The new preparation method was found to be comparable to the former one in term of in vivo efficacy. In another study, PLGA microparticles modified with $C T A B$ were found to have negative or positive charge dependent on the polymer used (molecular weight and hydrophobicity) [11]. In the same study, CTAB-PLGA microparticles were more toxic and less effective as gene carrier than polyethylenimine (PEI)-PLGA microparticles.

As shown above, the macromolecule chitosan and the small molecule CTAB can be used to modify PLGA particles for gene delivery but different results may be obtained from each one. It is not feasible to compare literature directly as each study used different polymers from different resources. Thus, to compare chitosan and CTAB, this study aimed to prepare PLGA nanoparticles modified with either CTAB or chitosan in total four formulations. Differential scanning calorimetry (DSC) and other characterisation techniques were employed to explore the differences in nanoparticle properties and their impact on the biological performance in vitro.

\section{EXPERIMENTAL}

\section{Materials}

PLGA5002A [ratio of lactic to glycolic acid is 50:50; $0.2 \mathrm{dl} / \mathrm{g}$ intrinsic viscosity (IV); acid terminated] was kindly provided by PURAC (Biochem, Netherland). Cetyltrimethylammonium bromide (CTAB), low molecular weight chitosan (LCS) of $50-190 \mathrm{kDa}$ and medium molecular weight chitosan (MCS) of 190-310 kDa were obtained from Sigma-Aldrich (Germany). Polyvinyl alcohol (PVA; MW 115 kDa, 88\% hydrolysed) was purchased from $\mathrm{BDH}$ Laboratory Supplies (Poole, England), 3-(4,5dimethylthiazol-2-yl)-2,5-diphenyltetrazolium

bromide (MTT) was purchased from Life Technologies (Grand Island, NY). Green fluorescent protein (GFP) Plasmid DNA (pDNA) was generously provided by Dr. Michael Bradbury (Mount Sinai School of Medicine, Department of Medicine, Manhattan, USA).

\section{Nanoparticle preparation}

Four formulations were prepared as listed in Table 1. CTAB and chitosan were used to modify PLGA nanoparticle surface in order to confer positive charge to the particles. Two of the formulations were modified with CTAB either in aqueous phase (F1) or in oil phase (F2). The other two formulations were modified by LCS (F3) or MCS (F4).

Table 1: Composition of nanoparticle formulations

\begin{tabular}{lllll}
\hline Phase/medium & F1 & F2 & F3 & F4 \\
\hline Oil phase & PLGA & PLGA & PLGA & PLGA \\
& DCM:EA ratio: & CTAB & DCM:EA ratio: & DCM:EA ratio: \\
& $1: 5$ & DCM:EA ratio: & $1: 5$ & $1: 5$ \\
& & $1: 1$ & & \\
Aqueous phase & PVA & PVA & PVA & PVA \\
& CTAB & Des & MCS \\
$\begin{array}{l}\text { Dispersion } \\
\text { medium }\end{array}$ & CTAB & Deionised water & LCS & MCS \\
\hline
\end{tabular}

All the materials were added at a concentration of $1 \% \mathrm{w} / \mathrm{v}$ 
Nanoparticle formulations were fabricated by modified emulsion solvent-diffusion method [3]. Briefly, the oily phase and the aqueous phase were homogenised for $3 \mathrm{~min}$ at 20,500 rpm using IKA® T10 homogeniser (IKA Werke $\mathrm{GmbH}$ and Co., Germany). The resulting emulsion was added to the dispersion medium and stirred for 2 $\mathrm{h}$ under vacuum to enable fast and complete evaporation of the organic solvents. Nanoparticles were centrifuged, washed three times then suspended in one millilitre of deionized water. The nanoparticles were lyophilized for DSC analysis or kept in suspension form in ice bath for zeta potential and other measurements.

\section{Differential scanning calorimetry (DSC)}

The DSC measurements were conducted on a differential scanning calorimeter (DSC1, STARe System, Mettler Toledo). Accurate weight of 2-3 $\mathrm{mg}$ of each nanoparticle formulation was put in a standard $40 \mu \mathrm{L}$ aluminium crucible with pin-hole. An empty pin-holed crucible was used as reference crucible. The thermal program was as follows: isothermal at $0{ }^{\circ} \mathrm{C}$ for $5 \mathrm{~min}$, heating from 0 to $85^{\circ} \mathrm{C}$, cooling to $-20^{\circ} \mathrm{C}$ then heated to $280{ }^{\circ} \mathrm{C}$. The thermal program for CTAB was: equilibrating for $5 \mathrm{~min}$ at $20^{\circ} \mathrm{C}$ then heating to $150{ }^{\circ} \mathrm{C}$. In all steps, purging with nitrogen gas and heating rate of $10^{\circ} \mathrm{C} / \mathrm{min}$ were used. The thermograms were processed using STARe Evaluation Software (Mettler Toledo AG).

\section{Zeta potential and conductivity measure- ments}

Nanoparticles were suspended in deionized water at $0.01 \% \mathrm{w} / \mathrm{v}$ prior to measurement of zeta potentials and conductivity by Zeta sizer Nano Z attached to a Malvern autotitrator MPT-2 (Malvern Instruments Ltd, Malvern, Worcestershire, UK).

\section{Fourier transform infrared spectroscopy (FTIR)}

Lyophilised nanoparticles were directly mixed with potassium bromide then the discs were prepared by compression. FTIR spectra were recorded in a Perkin-Elmer FTIR spectrometer (Perkin Elmer Corp., Norwalk, CT, USA) in the range of $400-4000 \mathrm{~cm}^{-1}$ with a resolution of 4 $\mathrm{cm}^{-1}$.

\section{Evaluation of cytotoxicity of nanoparticles}

Neuro 2a (N2a) cells were seeded in 96-well flatbottom microplate at a density of $2.5 \times 10^{5}$ cells per well and incubated for $48 \mathrm{~h}$ at $37^{\circ} \mathrm{C}$ and $5 \%$
$\mathrm{CO}_{2}$. After that, nanoparticles of the four formulations were added to it at different concentrations. Following $48 \mathrm{~h}$ incubation, the media were substituted with MTT reagent (30 $\mu \mathrm{L})$. After $4 \mathrm{~h}$ incubation, DMSO $(120 \mu \mathrm{L})$ was added. The absorbance was recorded at $570 \mathrm{~nm}$ using Tecan Infinite 200 microplate reader (Tecan Austria GmbH, Grödig, Austria). The viability of the cells were expressed as percentage relative to the control cells (untreated) which was considered as $100 \%$ viable.

\section{Assessment of cell uptake of nanoparticles by $\mathrm{N} 2 \mathrm{a}$ cells}

Coumarin-6 was added to the nanoparticle formulations as a fluorescent probe. Cell uptake study was conducted as we described before [12]. N2a cells at a density of $2.5 \times 10^{5}$ cells per well were incubated for $48 \mathrm{~h}$ at $37 \stackrel{\circ}{\circ} \mathrm{C}$ and $5 \%$ $\mathrm{CO}_{2}$ in a 96-well white flat-bottom microplate. Nanoparticles at concentration of $50 \mu \mathrm{g} / \mathrm{mL}$ were added to the cells. After $1 \mathrm{~h}$ incubation, cells were washed with ice-cold PBS three times to remove the non-internalised nanoparticles then visualised under an Olympus FSX100 fluorescence inverted microscope (Olympus, Tokyo, Japan). To extract and quantify coumarine-6, cells were lysed with lysis buffer (Promega; Madison, WI) then freeze-dried. The fluorescent dye Coumarin-6 was extracted with methanol and the fluorescence intensity was measured by fluorescence spectrophotometer (Perkin Elmer Corp., Norwalk, CT, USA). The excitation wave length $450 \mathrm{~nm}$ and the emission wave length $505 \mathrm{~nm}$ were used for the fluorescence measurement.

\section{Transfection efficiency}

Nanoparticles: pDNA (NP-pDNA) at ratio 20:1 (w:w) were mixed to make nanoparticle-pDNA (NP-pDNA) complexes and used to transfect N2a cells. Cells were cultured at $25 \times 10^{5}$ cells per well in a 24-well flat-bottom microplate and incubated for $48 \mathrm{~h}$ at $37 \stackrel{\circ}{\circ} \mathrm{C}$ and $5 \% \mathrm{CO}_{2}$. After that the media were replaced with fresh media containing NP-pDNA complexes and incubated for $48 \mathrm{~h}$. Cells were visualised by Nikon Eclipse Ti fluorescence microscope and the captured images were analysed with ImageJ (NIH, Bethesda, MD, USA) to quantify the green colour produced by GFP.

\section{Statistical analysis}

Comparison amongst the four formulations were done by analysis of variance (ANOVA) with Tukey's post hoc. T-test was employed for 
comparison within each formulation. $P<0.05$ was considered statistically significant. Minitab software version 16 (Minitab, State College, PA) was used for the statistical analysis.

\section{RESULTS}

\section{Thermal characteristics of nanoparticles}

The first heating of F1 showed a wide endothermic peak $\left(15.0-54.3^{\circ} \mathrm{C}\right)$ clearly different from the glass transition in F2 (Figure. $1)$. The $T_{g}$ of $\mathrm{F} 1$ and $\mathrm{F} 2$ was higher than $T_{g}$ of PLGA alone (Table 2). However, it is more ideal to compare with the formulation prepared by PLGA in the oil phase and PVA in the aqueous phase (i.e., the blank formulation). The $T_{g}$ of $\mathrm{F} 1$ and F2 was also higher than $T_{g}$ of blank formulation. The $T_{g}$ of F2 was slightly higher than the $T_{g}$ of $\mathrm{F} 1\left(43.8^{\circ} \mathrm{C}\right.$ compared to $43.3^{\circ} \mathrm{C}$, Table 2). The endothermic peak of $C T A B$ around $102^{\circ} \mathrm{C}$ was seen in $\mathrm{F} 1$ but not in $\mathrm{F} 2$. $\mathrm{F} 4$ exhibited significantly higher $T_{g}\left(46.7^{\circ} \mathrm{C}\right)$ as compared to
F3 $\left(42.5^{\circ} \mathrm{C}\right)$ (Figure. 2) and both were higher than the $T_{g}$ of the blank formulation (Table 2).

\section{Nanoparticle physicochemical characteri- sation: zeta potential, conductivity and FTIR}

CTAB-modified nanoparticles (F1 and F2) exhibited negative charge with lower value in F2 (Table 3). On the other hand, chitosan-modified nanoparticles (F3 and F4) were positively charged with higher zeta potential in LCSmodified particle (F3). Conductivity measurement (Table 3) revealed that $\mathrm{F} 1$ and $\mathrm{F} 2$ had higher conductivity than F3 and F4. No significant difference was seen between zeta potential values of F1 and F2 after lyophilisation and both suffered zeta potential inversion after lyophilisation. On the other hand, chitosanmodified nanoparticles remain positively charged after lyophilisation but $\mathrm{F} 3$ zeta potential decreased while F4 increased. Conductivity measurement (Table 3 ) revealed that CTABPLGA nanoparticles (F1 and F2) had higher conductivity than chitosan-PLGA nanoparticles (F3 and F4).

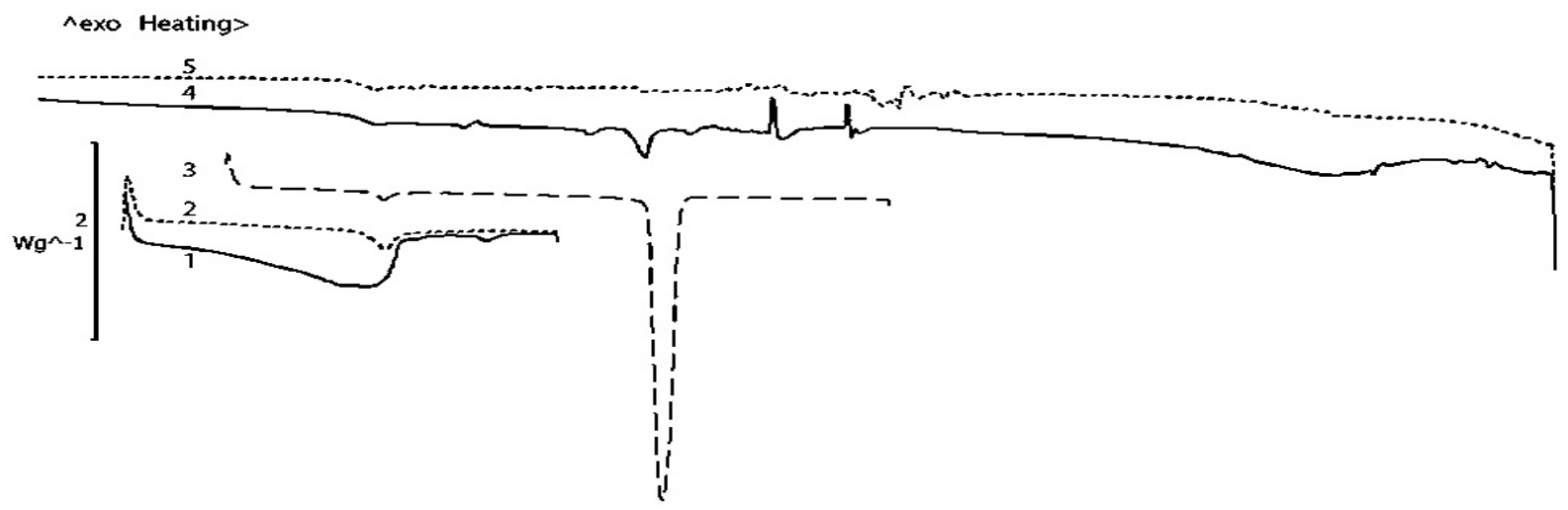

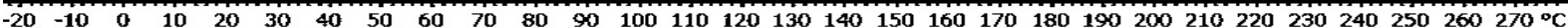

Figure 1: DSC thermograms for F1 (CTAB in aqueous phase) and F2 (CTAB in oil phase). (1) F1 first heating segment; (2) F2 first heating segment; (3) CTAB alone heating segment; (4) F1 second heating segment; (5) F2 second heating segment

Table 2: Glass transition of PLGA in different blends and formulations

\begin{tabular}{lcc}
\hline Sample & $\boldsymbol{T}_{\boldsymbol{g}}$ in first heating segment & $\begin{array}{c}\boldsymbol{T}_{\boldsymbol{g}} \text { in second heating } \\
\text { segment }^{* *}\end{array}$ \\
\hline PLGA & $41.9 \pm 0.07(\mathrm{C})$ & $37.6 \pm 0.10(\alpha)$ \\
PLGA-PVA physical mixture & $37.9 \pm 0.10(\mathrm{G})$ & $40.2 \pm 0.11(\beta)$ \\
PLGA-LCS physical mixture & $40.5 \pm 0.17(\mathrm{E})$ & $39.9 \pm 0.09(\mathrm{\gamma})$ \\
PLGA-MCS physical mixture & $42.1 \pm 0.25(\mathrm{~B}, \mathrm{C})$ & $39.4 \pm 0.15(\delta)$ \\
F1 & Not possible due to overlapping of enthalpy & $43.3 \pm 0.04(\varepsilon)$ \\
F2 & relaxation peak & \\
F3 & $47.0 \pm 0.07(\mathrm{~A})$ & $43.8 \pm 0.06(\zeta)$ \\
F4 & $38.8 \pm 0.06(\mathrm{~F})$ & $42.5 \pm 0.08(\eta)$ \\
Blank particles & $42.3 \pm 0.11(\mathrm{~B})$ & $46.7 \pm 0.27(\theta)$ \\
\hline
\end{tabular}

Blank particles were prepared by solely PLGA in oil phase and polyvinyl alcohol (PVA) in aqueous phase without further surface modification. ${ }^{* *}$ Mean of triplicate in each column in the table were compared by one-way ANOVA with Tukey post-hoc test. Means that do not share a letter are significantly different $(p<0.05)$ 


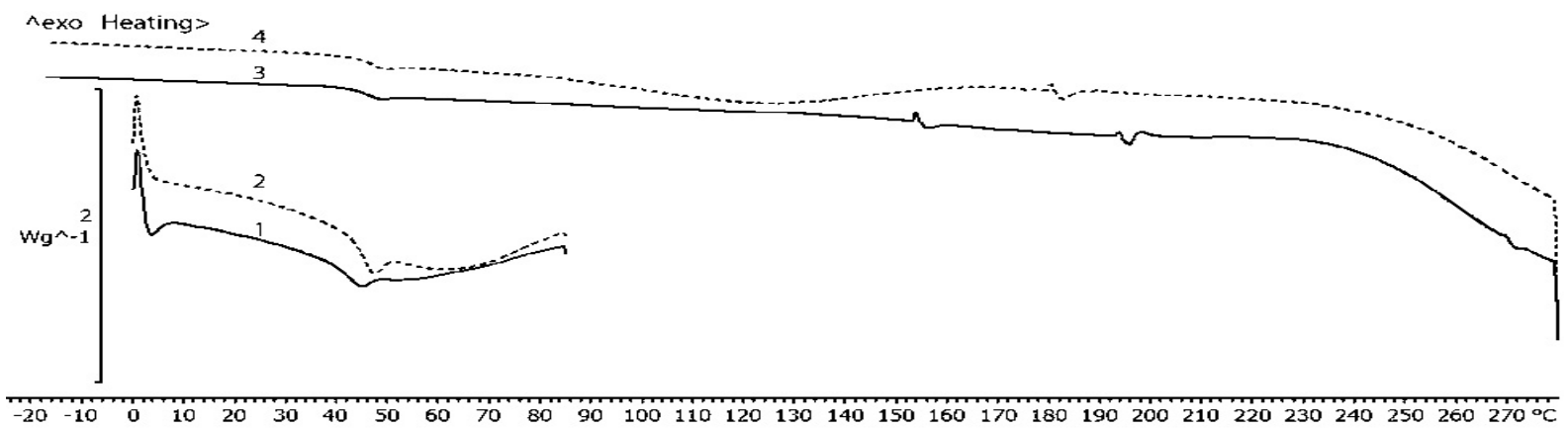

Figure 2: DSC thermograms for F3 (LCS-PLGA nanoparticle) and F4 (MCS-PLGA nanoparticle). (1) F3 first heating segment; (2) F4 first heating segment; (3) F3 second heating segment; (4) F4 second heating segment.

Table 3: Zeta potential and conductivity of nanoparticles suspended in deionized water (1 mg/10 mL). Formulation F1 was measured in three conditions: after normal washing step like other formulations ( 3 times washing with deionised water), without washing and extensive washing (washing 5 times with deionised water).

\begin{tabular}{lcccc}
\hline Formulation & & Zeta potential $(\mathbf{m V})$ & Conductivity $(\boldsymbol{\mu S} / \mathbf{c m})$ \\
\hline & Before lyophilisation & After lyophilisation & NP-pDNA complex & \\
\hline F1 & & & & \\
- without washing & $44.07 \pm 1.65^{(\mathrm{b})}$ & $24.80 \pm 3.32^{(\mathrm{a}, \mathrm{b})}$ & $-17.83 \pm 3.40^{(\mathrm{c})}$ & $23.77 \pm 0.06^{(\mathrm{a})}$ \\
- extensive washing & $-19.40 \pm 2.31^{(\mathrm{c})}$ & & & 300.0 \\
F2 & $-21.43 \pm 1.46^{(\mathrm{c})}$ & $19.57 \pm 11.64^{(\mathrm{a}, \mathrm{b})}$ & $-26.90 \pm 1.76^{(\mathrm{a})}$ & $3.26 \pm 1.68^{(\mathrm{b})}$ \\
F3 & $38.80 \pm 1.32^{(\mathrm{d})}$ & $10.47 \pm 0.25^{(\mathrm{b})}$ & $17.50 \pm 2.46^{(\mathrm{a})}$ & $12.79 \pm 6.44^{(\mathrm{a})}$ \\
F4 & $14.70 \pm 0.89^{(\mathrm{e})}$ & $31.00 \pm 4.85^{(\mathrm{a})}$ & $7.35 \pm 0.20^{(\mathrm{b})}$ & $5.45 \pm 0.10^{(\mathrm{b}, \mathrm{d})}$ \\
\hline
\end{tabular}

Data were represented as means of triplicate measurements; in each column means that do not share a letter are significantly different $(p<0.05)$.

FTIR spectra for the nanoparticle formulations are shown in Figure 3. PLGA exhibited characteristic bands at $1761 \mathrm{~cm}^{-1}$ (ester group) and 2953 and $2998 \mathrm{~cm}^{-}$(the axial stretching of sp2 and sp3 carbons)[6] which appeared at. $\mathrm{CTAB}$ exhibited several peaks, amongst them the -(CH2)- anti-symmetrical stretching at 2918 $\mathrm{cm}^{-1}$ and the symmetrical stretching at $2850 \mathrm{~cm}^{-1}$ [13] are the most interesting in this study. Chitosan exhibited the characteristic bands of $\mathrm{NH}_{2}$ scissoring vibration at $1640 \mathrm{~cm}^{-1}$ and $\mathrm{C}-\mathrm{O}$ stretching vibrations of the pyranose ring at 1075 and $1031 \mathrm{~cm}^{-1}[14,15]$. In all formulations, the characteristic peaks for PLGA appeared clearly in the FTIR spectra. In the case of F1 (CTAB in aqueous phase), the two peaks from $C T A B$ at 2920 and $2852 \mathrm{~cm}^{-1}$ were distinguishable in the spectrum. However, these peaks were not detected in F2. Chitosan peaks had low absorption and seem to be overlapped by PLGA peaks in $\mathrm{F} 3$ and $\mathrm{F} 4$. The increase in height of the peak at $1636 \mathrm{~cm}^{-1}$ and the small shoulder indicates chitosan presence was clearer in F4.

\section{Nanoparticle biological characteristics}

\section{Cytotoxicity of nanoparticles}

F1 revealed the highest cytotoxicity as shown in Figure 4. On the other hand, despite the suggested higher amount of CTAB molecules in
F2 but their presence in PLGA matrix may reduce the toxicity of this formulation. Chitosanmodified nanoparticles (F3 and F4) exhibited relatively low cytotoxicity. The cytotoxicity profile was similar between F3 and F4 with slightly higher cytotoxicity observed with F3.

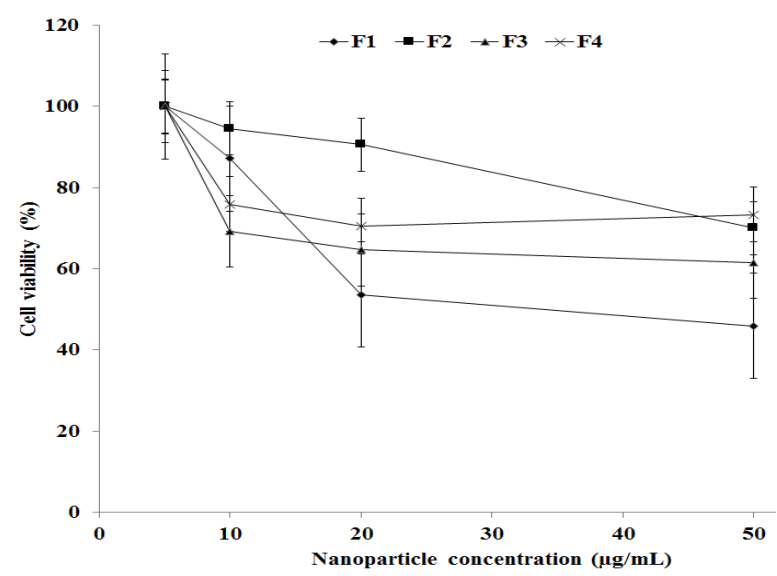

Figure 4: Cytotoxicity of the four nanoparticle formulations on N2a cells by MTT assay.

\section{Cell uptake of nanoparticles by N2a cells}

Chitosan-modified nanoparticles were taken up more than CTAB-modified nanoparticles (Figure 5a). Fluorescence inverted microscope images showed that almost all the cells in the field 

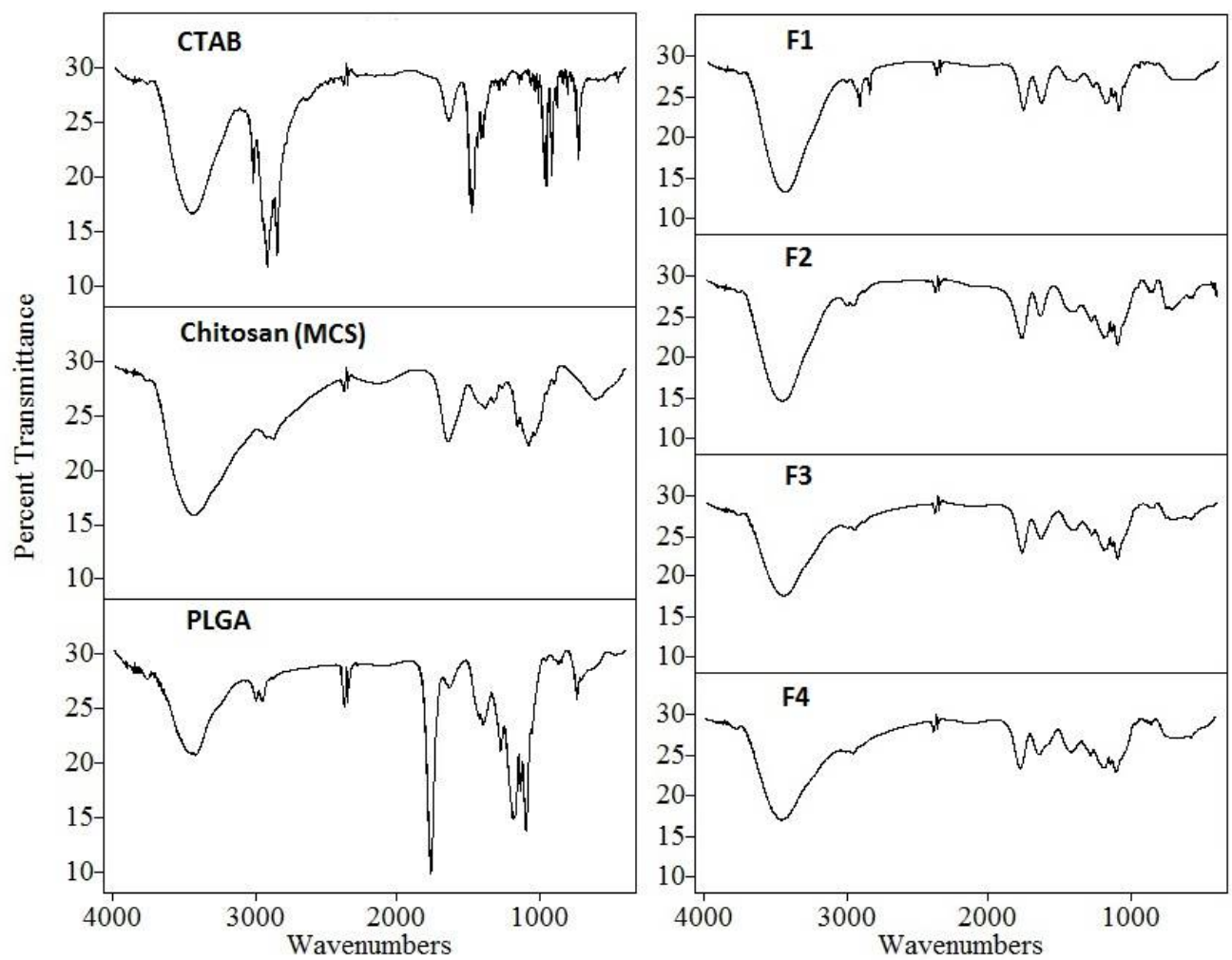

Figure 3: FTIR spectra of PLGA, CTAB, MCS and lyophilised nanoparticles

(a)

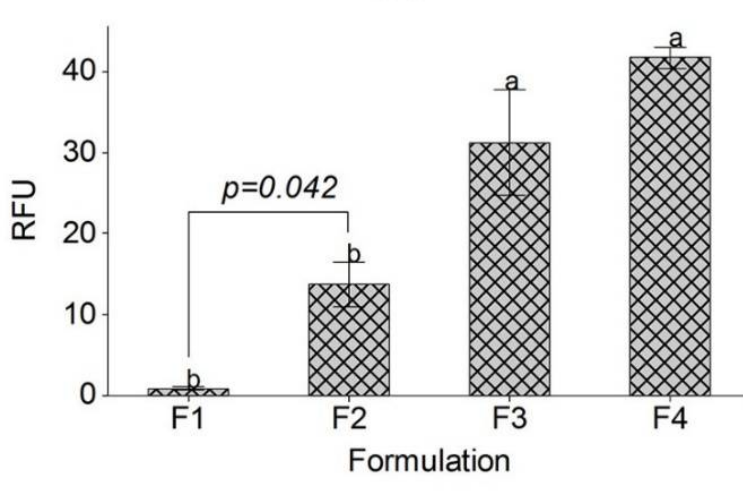

(b)
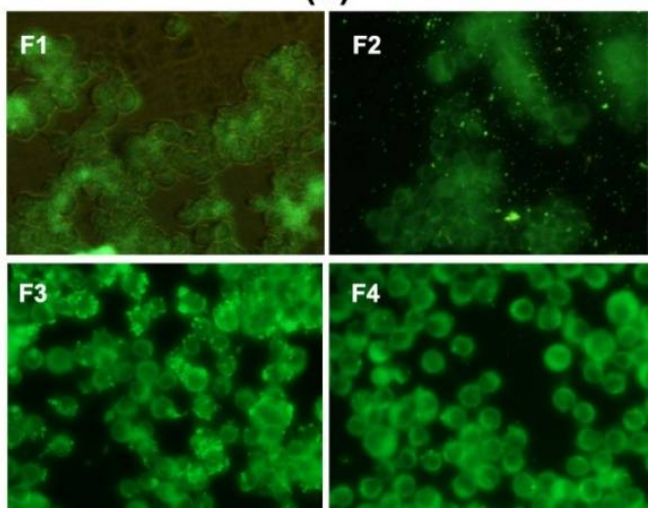

Figure 5: N2a cell uptake of coumarine-6 loaded nanoparticles as quantified by fluorimeter (a) and seen by fluorescence inverted microscope (b). In Figure 5(a), results were represented as means of triplicate measurements; letters over the bars represent the significant difference $(p<0.05$, ANOVA with Tukey post-hoc test)

successfully took up the nanoparticles during $1 \mathrm{~h}$ of incubation.

In the case of $\mathrm{F} 2$ that can be considered surfaceunmodified nanoparticles, free nanoparticles were observed in the field. In F3, some nanoparticles can be seen outside but stuck to the cells.

\section{Transfection of N2a cells}

Particles loaded with pDNA (encoding GFP) by adsorption were used to transfect N2a cells.
Cells were viewed under the fluorescence inverted microscope after $48 \mathrm{~h}$ to observe any expressed GFP (Figure 6a). The captured images were then analysed by ImageJ software (NIH, Bethesda, MD, USA) to compare the gene expression amongst the samples (Figure 6b). Image processing was based on the green coloured area which represents the number of transfected cells. Despite the negative zeta potential of F2, it exhibited higher transfection than F1 that almost did not show any gene expression. On the other hand, chitosan-modified 
(a)

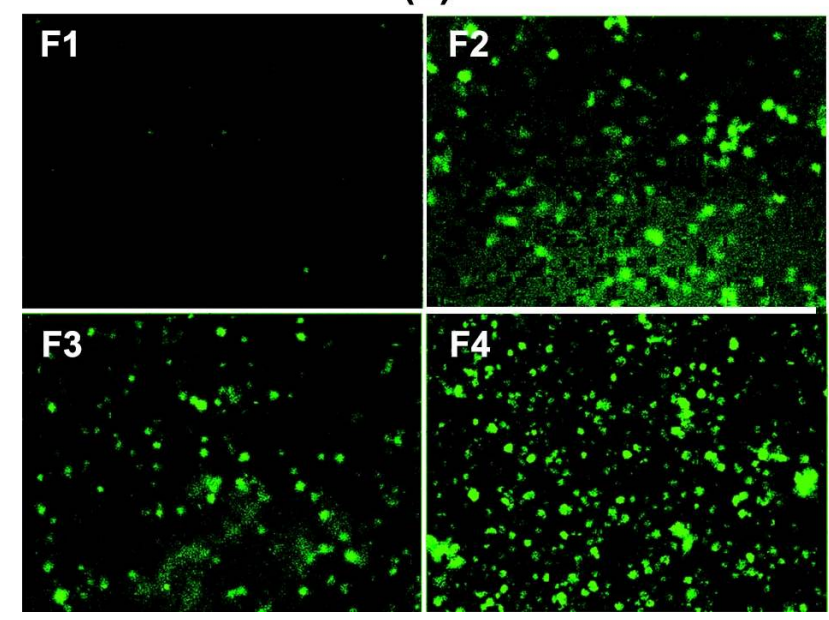

(b)

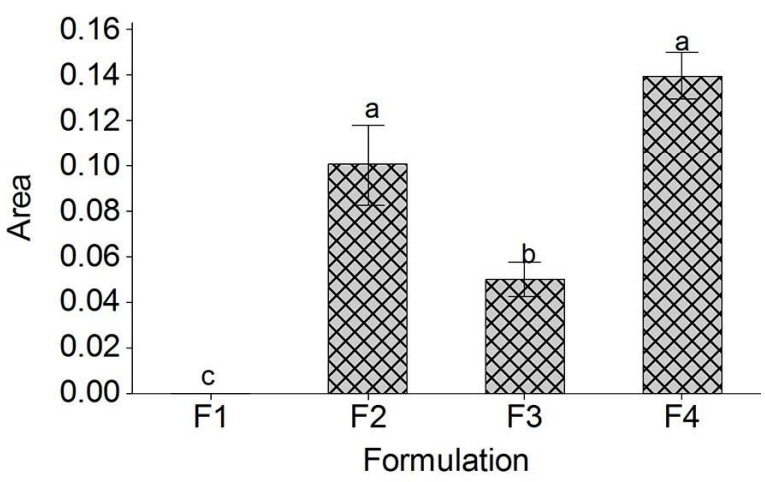

Figure 6: Transfection of N2a with pDNA (encoding GFP)-loaded nanoparticles as seen by fluorescence inverted microscope (a) and after image processing of the green colour area (b). In Figure 6(b), results were represented as means of triplicate measurements; letters over the bars represent the significant difference $(p<0.05$, ANOVA with Tukey post-hoc test)

nanoparticles exhibited different transfection efficiencies dependent on the molecular weight.

\section{DISCUSSION}

The low moisture content in the glassy material (PLGA) caused the wide endothermic peak (15.0 $-54.3^{\circ} \mathrm{C}$, Figure 1) in formulation $\mathrm{F} 1$ [16], which upon heating resulted in structural or enthalpy relaxation. The relaxation usually interferes with the $T_{g}$ measurement as the thermal event takes place near the $T_{g}$. The obvious difference in the thermograms of $\mathrm{F} 1$ and $\mathrm{F} 2$ near $T_{g}$ in the first heating segment suggests that nanoparticle micro-structures and properties differ if CTAB was added to the aqueous phase or oil phase. It was reported that CTAB adsorption on PLGA microparticles was mainly due to the hydrophobic and other weak interactions of the carbon chain with the dissolved PLGA [17]. However, CTAB has positive charge and thus it is assumed to have an ionic interaction with the negatively charged terminal carboxyl groups in the PLGA molecules. The polymer used in this study is PLGA with low molecular weight and free terminal carboxyl group. Consequently, the interaction between CTAB and PLGA might be stronger than previous studies. This interaction restricts PLGA molecule mobility leading to the observed higher $T_{g}$. Consequently, CTAB had anti-plasticising effect. This finding is opposite to what reported before that $\mathrm{CTAB}$ has plasticising effect on PLGA [11].

CTAB fractionates between the two aqueous and oil phases during the fabrication. When we used ethyl acetate and vacuum to facilitate the solvent diffusion and evaporation, the fast solidification of the nanoparticle caused CTAB molecules to remain in the oil phase in $\mathrm{F} 2$ and vice versa in $\mathrm{F} 1$. Therefore, CTAB content of the nanoparticles is larger in F2 compared to $\mathrm{F} 1$ and as a result, the $T_{g}$ of $\mathrm{F} 2$ was slightly higher than the $T_{g}$ of $\mathrm{F} 1$ $\left(43.8^{\circ} \mathrm{C}\right.$ compared to $\left.43.3^{\circ} \mathrm{C}\right)$.

At room temperature, CTAB crystal belongs to the monoclinic system, being optically anisotropic and it undergoes a phase transition at about 102 ${ }^{\circ} \mathrm{C}$ into an isotropic mesophase on heating [18]. The transition can be clearly seen in the second heating segment of F1 (Figure 1) but not of F2. This indicates the presence of CTAB, or at least a portion of it, in its crystalline form in $\mathrm{F} 1$. The presence of the crystalline phase of CTAB in F1 helped to conclude that significant amount of CTAB was adsorbed on the surface of $F 1$ but unlikely in the F2.

The significantly greater increase in the $T_{g}$ of F3 and F4 than that of the physical mixture of PLGA-CS indicates that chitosan has antiplasticising effect. Chitosan molecules anchored among PLGA molecules and greatly restricted their mobility. This also explains the stability of chitosan molecules during washing step, unlike CTAB molecules that were prone to desorption. Due to the larger molecules of MCS (190 - 310 kDa compared to 50 - $190 \mathrm{kDa}$ for LCS), MCS seems to have stronger effect on the PLGA glass transition than LCS. This means that MCS has stronger interaction with PLGA compared to LCS. The small thermal events appeared after $150^{\circ} \mathrm{C}$ in both $\mathrm{F} 3$ and $\mathrm{F} 4$ may be related to the movement of chitosan molecules because chitosan can be adsorbed on the PLGA particle in different conformations [19]. 
Contrary to several studies that reported positively charged particles whenever CTAB was included in the preparation $[5,20]$, this study revealed negatively charged particles with CTAB. One study reported negatively charged nanoparticle from CTAB-modified nanoparticles which was further demonstrated to be dependent on the type of PLGA and due to CTAB desorption from the particles in the washing step [11]. This concept was exploited by Singh, Ugozzoli [9] whereby the washing steps were controlled to obtain different concentrations of CTAB in PLGA microparticle. Oster et al [11] proposed the formation of reverse micelles where the orientation of the hydrophilic, ionised head groups of CTAB molecules tend to converge inwards the core of the micelle masking the positive charges. The desorption of CTAB molecules from the particles increased the conductivity of CTAB-PLGA nanoparticles. This was supported by the dramatic decreased in conductivity of the particles subjected to extensive washing cycles.

Furthermore, CTAB molecules suffer relocalisation and re-orientation during lyophilisation. It seems that during the lyophilisation cycle then re-suspending the particles in deionised water, CTAB molecules migrated to the surface of the particles orienting their polar head to the surface rather than arranging themselves in a reversed micelle conformation. This led to the zeta potential inversion of $\mathrm{F} 1$ and F2. In contrast, DSC measurements suggest that chitosan strongly interacts with the polymeric network of PLGA matrix. This reflects the significantly lower conductivity reading of $\mathrm{F} 3$ and $\mathrm{F} 4$ compared to $\mathrm{F} 1$ and $\mathrm{F} 2$ (Table 3). In addition, the strong interaction of MCS molecules with PLGA, as revealed by DSC, results in presenting less amine groups to the particle surface and this explains the lower zeta potential of F4 compared to F3.

Cationic polymers have often been demonstrated to cause membrane toxicity as a result of electrostatic interactions with the negatively charged glycocalyx of the cellular surface $[21,22]$. Both CTAB and chitosan solutions were also reported to be toxic to the cells $[11,23]$. The presence of CTAB molecules on the surface of F1 nanoparticles and their readily desorption from to the solution give rise to the high toxicity. On the other hand, despite the suggested higher amount of CTAB molecules in F2, their presence in PLGA matrix may reduce the toxicity of this formulation. Chitosan-modified nanoparticles (F3 and F4) exhibited relatively low cytotoxicity which can be attributed to the inherited low toxicity of chitosan compared to CTAB and the tight adhesion of chitosan molecules to the particles.

The very low cell uptake observed in $\mathrm{F} 1$ may be related to its cytotoxicity as CTAB readily desorb from the surface of the particles. The presence of some particles in the field could be attributed to the exocytosis. Panyam and Labhasetwar [24] demonstrated that the amount of nanoparticles within the cell was maintained as long as the nanoparticles remained in the external medium and when the external nanoparticle concentration gradient was removed, nanoparticle exocytosis started to take place. The presence of free nanoparticles in the case of surface-unmodified nanoparticles highlights the significance of surface modification in the cell uptake. Chitosan molecules entangle in PLGA matrix leaving portion exposed to the solution facilitating the nanoparticle-cell interaction.

The low cell uptake of $\mathrm{F} 1$ and the instability of the complex with pDNA due to CTAB desorption from the particles result in low transfection. Zeta potential of F3-pDNA was higher than F4-pDNA (Table 3), which may result in better interaction with the cells and hence higher transfection efficiency. The unexpected high transfection observed by $\mathrm{F} 2$ may be attributed to the weak interaction with pDNA with the aid of CTAB molecules in the nanoparticle matrix with their positive head towards the surface. These CTAB molecules are bound strongly to PLGA in F2, unlike in $\mathrm{F} 1$. This interaction may be sufficient to carry the pDNA into the cell then readily dissociate it from the nanoparticles. Similar results were obtained by Köping-Höggård, Vårum [25] who showed that polyplexes with easier dissociation could mediate faster effect and thus higher gene expression.

Based on the characterisation results, schematic diagrams for the four formulations were drawn as shown in Figure 7. F1 is characterised by the presence of CTAB in PLGA matrix in the reverse micelle configuration converging the ionised head groups inwards the core of the micelle. $C T A B$ is also adsorbed on the surface with partial coverage and crystallises after lyophilisation. The overall surface charge is negative and $C T A B$ is prone to desorption from the particles. On the other hand, F2 is characterised by the presence of CTAB mainly in the PLGA matrix in the reverse micelle configuration. The surface charge was also negative but lower than $\mathrm{F} 1$ and CTAB is prone to desorb slowly from the particles. F3 and F4 are characterised by the presence of chitosan in PLGA matrix which completely covered the nanoparticle surface. 


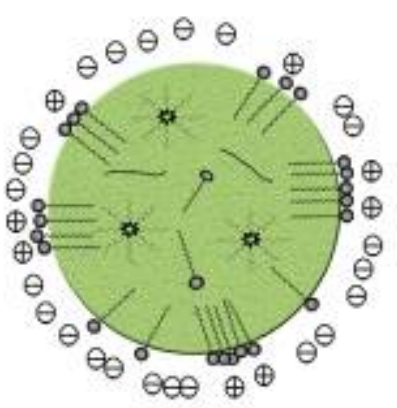

F1

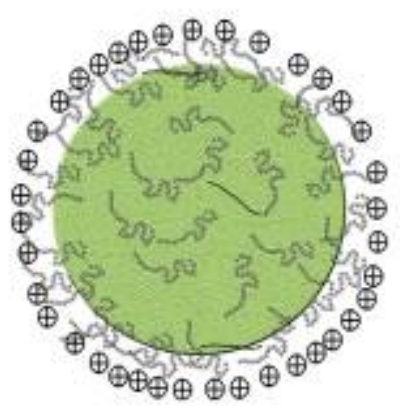

F3

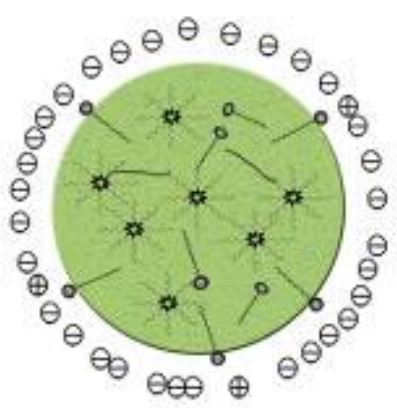

F2

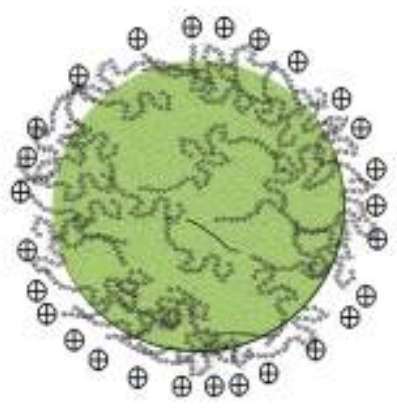

F4

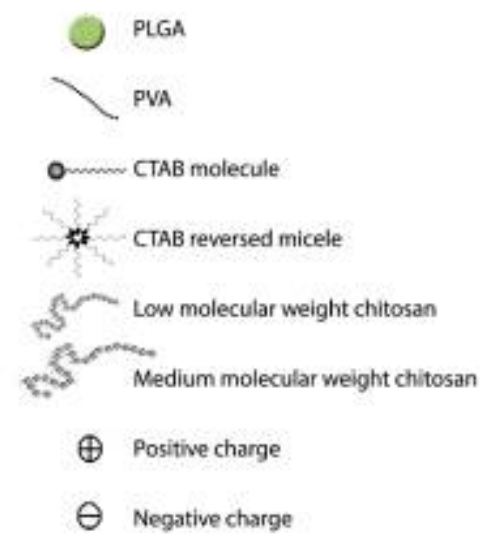

Figure 7: Proposed schematic diagrams based on DSC, zeta potential and conductivity results for (1) F1 (PLGA nanoparticles modified with CTAB in aqueous phase), (2) F2 (PLGA nanoparticles modified with CTAB in oil phase), (3) F3 (PLGA nanoparticles modified with low molecular weight chitosan and (4) F4 (PLGA nanoparticles modified with medium molecular weight chitosan).

In addition, chitosan interacts stronger with PLGA in F4 than in F3, however, in both formulations chitosan is stable in the particles and not prone to desorption. This yielded positively charged surface, lower cytotoxicity and higher cell uptake than CTAB-PLGA nanoparticles.

\section{CONCLUSION}

PLGA nanoparticles modified with CTAB or chitosan have successfully been prepared. Chitosan as a cationic modifier for PLGA nanoparticles is superior to CTAB in terms of producing particles that have positive zeta potential, low cytotoxicity, and high cell uptake and transfection. Chitosan-modified nanoparticles are more effective for plasmid DNA adsorption and protection. This results suggest that chitosan-modified PLGA nanoparticles can be developed as a plasmid DNA carrier for gene therapy.

\section{DECLARATIONS}

\section{Acknowledgement}

This work was funded by IIUM Research Initiative Grant Scheme (Gran ID: RIGS16-325-
0489) and Ministry of Science, Technology \& Innovation of Malaysia (MOSTI) (Grant id: 02-0108-SF0101).

\section{Conflict of Interest}

No conflict of interest associated with this work.

\section{Contribution of Authors}

The authors declare that this work was done by the authors named in this article and all liabilities pertaining to claims relating to the content of this article will be borne by them.

\section{Open Access}

This is an Open Access article that uses a funding model which does not charge readers or their institutions for access and distributed under the terms of the Creative Commons Attribution License (http://creativecommons.org/licenses/by/ 4.0) and the Budapest Open Access Initiative (http://www.budapestopenaccessinitiative.org/rea d), which permit unrestricted use, distribution, and reproduction in any medium, provided the original work is properly credited. 


\section{REFERENCES}

1. Bala I, Hariharan S, Kumar M. PLGA nanoparticles in drug delivery: the state of the art. Crit Rev Ther Drug Carrier Syst 2004; 21(5): 387-422.

2. Doolaanea AA, Ismail AFH, Mansor NI, Nor NHM, Mohamed F. Effect of Surfactants on Plasmid DNA Stability and Release from Poly (D, L-lactide-coglycolide) Microspheres. Trop J Pharm Res 2015; 14(10): 1769-1778.

3. Doolaanea AA, Mansor NI, Mohd Nor NH, Mohamed F. Co-encapsulation of Nigella sativa oil and plasmid DNA for enhanced gene therapy of Alzheimer's disease. J Microencapsul 2016; 33(2): 114-126.

4. Yue $Y$, Jin F, Deng R, Cai J, Dai Z, Lin MCM, Kung H-F, Mattebjerg MA, Andresen TL, Wu C. Revisit complexation between DNA and polyethylenimine -Effect of length of free polycationic chains on gene transfection. J Control Release 2011; 152(1): 143-151.

5. Basarkar A, Devineni D, Palaniappan R, Singh J. Preparation, characterization, cytotoxicity and transfection efficiency of poly(dl-lactide-co-glycolide) and poly(dl-lactic acid) cationic nanoparticles for controlled delivery of plasmid DNA. Int J Pharm 2007; 343(1-2): 247-254.

6. Ravi Kumar M, Bakowsky U, Lehr C. Preparation and characterization of cationic PLGA nanospheres as DNA carriers. Biomaterials 2004; 25(10): 1771-1777.

7. Kim BS, Kim CS, Lee KM. The intracellular uptake ability of chitosan-coated Poly (D,L-lactideco-glycolide) nanoparticles. Arch Pharm Res 2008; 31(8): 1050-1054.

8. Bordelon H, Biris AS, Sabliov CM, Monroe WT. Characterization of plasmid DNA location within chitosan/PLGA/pDNA nanoparticle complexes designed for gene delivery. J Nanomater 2011; 2011: 1-8.

9. Singh M, Ugozzoli M, Briones M, Kazzaz J, Soenawan E, O'Hagan DT. The effect of CTAB concentration in cationic PLG microparticles on DNA adsorption and in vivo performance. Pharm Res 2003; 20(2): 247-251.

10. Singh $M$, Fang JH, Kazzaz J, Ugozzoli M, Chesko J, Malyala $P$, Dhaliwal $R$, Wei $R$, Hora M, O'Hagan D. A modified process for preparing cationic polylactide-coglycolide microparticles with adsorbed DNA. Int J Pharm 2006; 327(1-2): 1-5.

11. Oster CG, Kim N, Grode L, Barbu-Tudoran L, Schaper AK, Kaufmann SHE, Kissel T. Cationic microparticles consisting of poly(lactide-co-glycolide) and polyethylenimine as carriers systems for parental DNA vaccination. J Control Release 2005; 104(2): 359-377.

12. Doolaanea AA, Mansor NI, Mohd Nor NH, Mohamed F. Cellular uptake of Nigella sativa oil-PLGA microparticle by PC-12 cell line. J Microencapsul 2014; 31(6): 600608.

13. Huang L, Chen X, Li Q. Synthesis of microporous molecular sieves by surfactant decomposition. J Mater Chem 2001; 11(2): 610-615

14. Liu W, Sun S, Cao Z, Zhang X, Yao K, Lu WW, Luk KDK. An investigation on the physicochemical properties of chitosan/DNA polyelectrolyte complexes. Biomaterials 2005; 26(15): 2705-2711.

15. Banerjee T, Mitra S, Kumar Singh A, Kumar Sharma R, Maitra A. Preparation, characterization and biodistribution of ultrafine chitosan nanoparticles. Int $\mathrm{J}$ Pharm 2002; 243(1-2): 93-105.

16. Chung H-J, Lee E-J, Lim S-T. Comparison in glass transition and enthalpy relaxation between native and gelatinized rice starches. Carbohydr Polym 2002; 48(3): 287-298.

17. Wischke C, Borchert H-H, Zimmermann J, Siebenbrodt I, Lorenzen DR. Stable cationic microparticles for enhanced model antigen delivery to dendritic cells. J Control Release 2006; 114(3): 359-368.

18. Endoh K, Suga $H$. Phase behavior of CTAB: oiodophenol binary system. Thermochim Acta 1999; 334(1-2): 89-96.

19. Guo C, Gemeinhart RA. Understanding the adsorption mechanism of chitosan onto poly(lactide-co-glycolide) particles. Eur J Pharm Biopharm 2008; 70(2): 597-604.

20. Zou W, Liu C, Chen Z, Zhang N. Preparation and Characterization of Cationic PLA-PEG Nanoparticles for Delivery of Plasmid DNA. Nanoscale Res Lett 2009; 4(9): 982-992.

21. Fischer D, Li Y, Ahlemeyer B, Krieglstein J, Kissel T. In vitro cytotoxicity testing of polycations: influence of polymer structure on cell viability and hemolysis. Biomaterials 2003; 24(7): 1121-1131.

22. Choksakulnimitr S, Masuda S, Tokuda H, Takakura Y, Hashida M. In vitro cytotoxicity of macromolecules in different cell culture systems. J Control Release 1995; 34(3): 233-241.

23. Tahara K, Yamamoto $H$, Kawashima Y. Cellular uptake mechanisms and intracellular distributions of polysorbate 80-modified poly (D,L-lactide-co-glycolide) nanospheres for gene delivery. Eur J Pharm Biopharm 2010; 75(2): 218-224.

24. Panyam J, Labhasetwar V. Biodegradable nanoparticles for drug and gene delivery to cells and tissue. Adv Drug Deliv Rev 2012; 64: 61-71.

25. Köping-Höggård $M$, Vårum $K$, Issa $M$, Danielsen $S$, Christensen B, Stokke B, Artursson P. Improved chitosan-mediated gene delivery based on easily dissociated chitosan polyplexes of highly defined chitosan oligomers. Gene Ther 2004; 11(19): 14411452. 